\title{
HARDY-LITTLEWOOD INEQUALITY FOR QUASIREGULAR MAPPINGS IN CERTAIN DOMAINS IN $\boldsymbol{R}^{n}$
}

\author{
T. IWANIEC and C. A. NOLDER
}

\section{Introduction}

In their paper "Some properties of conjugate functions" [7] Hardy and Littlewood proved the following

Theorem 1. If $f=u+i v$ is analytic in a disk $D \subset \boldsymbol{R}^{2}$ centered at $z_{0}$, then

$$
\int_{D}\left|v(z)-v\left(z_{0}\right)\right|^{p} d x d y \leqq C_{p} \int_{D}\left|u(z)-u\left(z_{0}\right)\right|^{p} d x d y
$$

for $0<p<\infty$, where $C_{p}$ does not depend on $f$.

Generalizations of this theorem to solutions of elliptic systems of P.D.E.'s in several variables as well as to more general domains were treated in a recent paper by J. Boman [2] for $1 \leqq p<\infty$; they can be extended to hold for $0<p<\infty$.

We will establish similar estimates for the components of a quasiregular mapping in domains in $\boldsymbol{R}^{n}$ which satisfy certain geometric conditions (see Theorem 4 and Corollaries $1-5$ ). The main idea we use is based on two geometric results. The first, Theorem 2, states that the exponent can be improved in a weak type reverse Hölder inequality. This should be considered as complementary to Gehring's well known Lemma [5]. The second, Theorem 3, allows us to obtain global estimates from the local inequalities over the cubes in the domain. Both Theorem 2 and Theorem 3 illustrate a self-improving property of some local estimates and they seem to be interesting in their own right. The local estimates (Propositions 1,2,3) are obtained from the classical embedding inequalities. We do not appeal to any nonstandard or difficult result from quasiconformal theory and differential equations.

The weighted inequalities as an application give us a version of Theorem 1 over a quasiball (see Corollary 5).

Proposition 4 explains the behaviour at infinity and near the boundary of the upper-half space $\mathrm{K}$ of a quasiregular mapping from $L^{p}(\mathrm{H})$.

This research was supported in part by the U.S. National Science Foundation, Grant MCS 82-01607. 
We express our gratitude to Professor F. W. Gehring for his constant guidance, insight, and encouragement while we were working on this paper.

\section{Notation and definitions}

We let $\boldsymbol{R}^{n}$ denote the real $n$-dimensional Euclidean space and $Q$ any open cubes with diameter diam $Q$ and volume $|Q|$. If $\sigma \geqq 1$, then $\sigma Q$ will be the cube with the same center as $Q$ but expanded by the factor $\sigma$. The symbol $d x$ will be Lebesgue measure on $\boldsymbol{R}^{n},|E|$ will be the measure of the set $E \subset \boldsymbol{R}^{n}$ and $\chi_{E}$ the characteristic function of $E$. We also work with absolutely continuous sigma additive measures $\mu$ on $\boldsymbol{R}^{n}, d \mu(x)=w(x) d x$, with the positive density $w$ from $L_{\text {loc }}^{1}\left(\boldsymbol{R}^{n}\right)$ and other classes. If $0<p<\infty$, we denote by $L^{p}(E, \mu)$ the space of all measurable functions $f$ with

$$
\|f\|_{p, E, \mu}=\left(\int_{E}|f(x)|^{p} d \mu(x)\right)^{1 / p}<\infty .
$$

The Minkowski type inequality

$$
\|f+g\|_{p, E, \mu} \leqq 2^{1 / p}\left(\|f\|_{p, E, \mu}+\|g\|_{p, E, \mu}\right)
$$

will be frequently used for $f, g \in L^{p}(E, \mu)$ with $p>0$. We also introduce the sharp $L^{p_{-} \text {"norms" }}$

$$
\|f\|_{p, E, \mu}^{\#}=\inf _{a \in R}\|f-a\|_{p, E, \mu}<\infty .
$$

Clearly, if $E_{1} \subset E_{2}$ then $\|f\|_{p, E_{1}, \mu}^{\#} \leqq\|f\|_{p, E_{2}, \mu}^{\#}$ and the infimums are attained. If $p \geqq 1$ and $0<\mu(E)<\infty$, we have

$$
\left\|f_{p, E, \mu}^{\#} \leqq\right\| f-f_{E, \mu}\left\|_{p, E, \mu} \leqq 2\right\| f^{\prime} \|_{p, E, \mu}^{\#},
$$

where $f_{E, \mu}$ is the $\mu$-average of $f$ on $E$

$$
f_{E, \mu}=\frac{1}{\mu(E)} \int_{E} f(x) d \mu(x)=f_{E} f d \mu .
$$

The following Fatou type lemma holds for the sharp norms.

Lemma 1. Given an ascending sequence of measurable sets $E_{1} \subset E_{2} \subset \ldots$, suppose $f \in L^{p}\left(E_{i}, \mu\right), \quad 0<p<\infty$ and $\sup _{i}\|f\|_{p, E_{i}, \mu}^{\#}<\infty$ for $i=1,2, \ldots$ Then $f \in L^{p}(E, \mu)$, where $E=\bigcup_{i=1}^{\infty} E_{i}$, and the sequence $\left\{\|f\|_{p, E_{i}, \mu}^{\#}\right\}_{i=1}^{\infty}$ increases to the limit $\|f\|_{p, E, \mu}^{\#}$.

Proof. We may assume that $0<\mu\left(E_{1}\right)<\infty$. For each $i \geqq 1$ we can find a number $a_{i} \in \boldsymbol{R}$ such that

Hence

$$
\left\|f-a_{i}\right\|_{p, E_{i}, \mu}=\|f\|_{p, E_{i}, \mu}^{\#} .
$$

$$
\begin{aligned}
\left|a_{i}\right|\left(\mu\left(E_{1}\right)\right)^{1 / p}= & \left(\int_{E_{1}}\left|a_{i}\right|^{p} d \mu\right)^{1 / p} \leqq 2^{1 / p}\left\|f-a_{i}\right\|_{p, E_{i}, \mu}+2^{1 / p}\|f\|_{p, E_{1}, \mu} \\
& \leqq 2^{1 / p} \sup _{l}\|f\|_{p, E_{l}, \mu}^{\#}+2^{1 / p}\|f\|_{p, E_{1}, \mu} .
\end{aligned}
$$


Now, as a bounded sequence, $\left\{a_{i}\right\}_{i=1}^{\infty}$ has a convergent subsequence, say $\lim _{v \rightarrow \infty} a_{i_{v}}=a$. By Fatou's lemma we get

$$
\|f-a\|_{p, E, \mu} \leqq \lim _{v \rightarrow \infty}\left\|f-a_{i_{v}}\right\|_{p, E_{i_{v}}, \mu}=\lim _{v \rightarrow \infty}\|f\|_{p, E_{i_{v}}, \mu}^{\#},
$$

which shows that $f-a \in L^{p}(E, \mu)$. Since the sequence $\left\{\|f\|_{p, E_{i}, \mu}^{\#}\right\}_{i=1}^{\infty}$ increases, we also conclude that $\|f\|_{p, E, \mu}^{\#}=\lim _{i \rightarrow \infty}\|f\|_{p, E_{i}, \mu}^{\#}$. Hereafter, for notational simplicity, the symbol $\mu$ will be omitted from the formulas when $d \mu(x)=d x$.

The set $\Omega$ will always be an open set in $\boldsymbol{R}^{n}$. We will deal with the following spaces of functions defined on $\Omega$ :

$C^{\infty}(\Omega)$ - the class of indefinitely differentiable functions,

$C_{0}^{\infty}(\Omega)$ - the subclass of $C^{\infty}(\Omega)$ of functions with compact support,

$W_{p}^{1}(\Omega), 1 \leqq p<\infty$, - Sobolev space which is the completion of $C^{\infty}(\Omega)$ with respect to the norm

$$
\|U\|_{W_{p}^{1}(\Omega)}=\|U\|_{p, \Omega}+\|\nabla U\|_{p, \Omega} .
$$

Here $\nabla=\left(\partial / \partial x_{1}, \ldots, \partial / \partial x_{n}\right)$ is the gradient operator. $W_{p, \text { loc }}^{1}(\Omega)=\bigcap_{\Omega^{\prime}} W_{p}^{1}\left(\Omega^{\prime}\right)$, where $\Omega^{\prime}$ is an arbitrary open set compactly contained in $\Omega$.

We will require the following version of the Poincaré and Sobolev inequalities.

Lemma 2. Let $Q$ be an open cube in $\boldsymbol{R}^{n}$ and let $U \in W_{s}^{1}(Q)$. If $s=n$, then $U \in L^{p}(Q)$ for each $p>0$ and

$$
\left(f_{Q}\left|U-U_{Q}\right|^{p}\right)^{1 / p} \leqq C_{1}(n, p)\left(f_{Q}|\nabla U|^{n}\right)^{1 / n}
$$

If $s>n$, then $U \in L^{\infty}(Q)$ and

$$
\|U\|_{\infty, Q} \leqq C_{2}(n, s)\left[\left(f_{Q}|U|^{s}\right)^{1 / s}+\operatorname{diam} Q\left(f_{Q}|\nabla U|^{s}\right)^{1 / s}\right] .
$$

These inequalities, for example, can be deduced from [6]; see formula (7.45) and problem 7.11 on page 164 . For $f \in L^{p}(\Omega, \mu), 0<p<\infty$, we let $M_{p}(f, \mu)$ denote the Hardy-Littlewood maximal function

$$
M_{p}(f, \mu)(x)=\sup \left\{\left(f_{Q} \mid f^{p} d \mu\right)^{1 / p} ; x \in Q \subset \Omega\right\}
$$

and $M_{p}^{\#}(f, \mu)$ the corresponding sharp maximal function

$$
M_{p}^{\#}(f, \mu)(x)=\sup \left\{\inf _{a \in R}\left(f_{Q}|f-a|^{p} d \mu\right)^{1 / p} ; x \in Q \subset \Omega\right\} .
$$

In both formulas the supremum is taken over all cubes $Q \subset \Omega$ containing the point $x$.

Next we introduce the BMO norm for $f \in L^{1}(\Omega, \mu)$ by

$$
\|f\|_{\Omega, \mu}^{\text {BMO }}=\sup _{x \in \Omega} M_{1}^{\#}(f, \mu)(x) .
$$

Inequality (2.1) shows that $\|f\|_{\Omega, \mu}^{\mathrm{BMO}}$ is equivalent to the usual BMO norm when $d \mu(x)=d x$. 
Finally we say that $w$ is a Muckenhoupt weight, $w \in A_{M}^{q}(\Omega)$, where $1<q<\infty$ and $1 \leqq M<\infty$, if $w>0$ and

$$
f_{Q} w(x) d x \leqq M\left(f_{Q} w(x)^{1 /(1-q)} d x\right)^{1-q}
$$

for each cube $Q \subset \Omega$ [10]. Such functions $w$ arise in many classical inequalities. Inequality (2.4) immediately implies the following property of the measure $d \mu(x)=$ $w(x) d x$ :

$$
\mu(\sigma Q) \leqq M \sigma^{n q} \mu(Q)
$$

for $\sigma>1$ and each cube $Q$ with $\sigma Q \subset \Omega$.

Notice that the Jacobian of a $K$-quasiconformal mapping in $\boldsymbol{R}^{n}$ belongs to $A_{M}^{q}\left(\boldsymbol{R}^{n}\right)$ for some $q=q(n, K)>1$ and $M=M(n, K) \geqq 1$. This follows from [3] and [5].

Inequality (2.4) also implies that

$$
f_{Q} w(x) d x \leqq M\left(f_{Q} \sqrt{w(x)} d x\right)^{2},
$$

which is a reverse Hölder inequality; consequently, we obtain the following

Lemma 3. Let $w \in A_{M}^{q}\left(\boldsymbol{R}^{n}\right), q>1, \quad M \geqq 1$. Then there exists an exponent $\beta=\beta(n, M)>1$ such that

(see [3], [5]).

$$
\left(f_{Q} w(x)^{\beta} d x\right)^{1 / \beta} \leqq C_{3}(n, M) f_{Q} w(x) d x
$$

\section{Improving the exponent in a reverse Hölder inequality}

Theorem 2. Let $0<s<p$ and $f \in L_{\mathrm{loc}}^{p}(\Omega)$. Suppose that for each cube $Q$ with $2 Q \subset \Omega$,

$$
\left(f_{Q}|f|^{p}\right)^{1 / p} \leqq A\left(f_{2 Q}|f|^{s}\right)^{1 / s}
$$

where $A$ is independent of $Q$. Then for each $r>0, \sigma>1$ and each cube $Q$ with $\sigma Q \subset \Omega$,

$$
\left(f_{Q}|f|^{p}\right)^{1 / p} \leqq B(\sigma)\left(f_{\sigma Q}|f|^{r}\right)^{1 / r},
$$

where $B(\sigma)$ depends only on $\sigma, n, p, s, r$ and $A$.

Proof. By Hölder's inequality we may assume $r<s<p$. Set $\beta=p(s-r) / s(p-r) \epsilon$ $(0,1)$ so that $1 / s=\beta / p+(1-\beta) / r$. Hölder's inequality and the assumption (3.0) imply that

$$
\left(f_{Q}|f|^{p}\right)^{1 / p} \leqq A\left(f_{2 Q}|f|^{p}\right)^{\beta / p}\left(f_{2 Q}|f|^{r}\right)^{(1-\beta) / r}
$$


for each cube $Q$ with $2 Q \subset \Omega$. Fix a cube $R \subset \Omega$, and denote by $R_{v}=\left(1-2^{-v}\right) R$, $R_{\infty}=R$ for $v=1,2, \ldots$. Next subdivide $R_{v}$ into $\left(2^{v+1}-2\right)^{n}$ nonoverlapping congruent closed cubes. Denote the family of these cubes by $F_{v}$. Next for every $v=1,2, \ldots$ define by induction the collection $\mathscr{F}_{v}$ of cubes from the union $\bigcup_{\alpha>1} F_{\alpha}$ as follows:

$$
\mathscr{F}_{1}=F_{1}, \quad \mathscr{F}_{v+1}=\mathscr{F}_{v} \cup\left\{Q \in F_{v+1} ; Q \nsubseteq R_{v}\right\} \quad \text { for } \quad v=1,2, \ldots
$$

Then by geometry we have:

i)

ii)

iii)

iv)

v)

$$
R_{k}=\bigcup_{Q \in \mathscr{F}_{k}} Q
$$

$$
R_{k+1}=\bigcup_{Q \in \mathscr{F}_{k}} 2 Q
$$

$$
\chi_{R_{k+1}} \leqq \sum_{Q \in \mathscr{F}_{k}} \chi_{2 Q} \leqq 4^{n} \chi_{R_{k+1}},
$$

$$
\left|R_{k+1}\right|=\left(1-2^{-k-1}\right)^{n}|R|=\left(\frac{3}{2}\right)^{n}\left|R_{k}\right| \text {, }
$$

$|Q| \geqq 2^{-n(k+1)}|R|$ for $Q \in \mathscr{F}_{k}$,

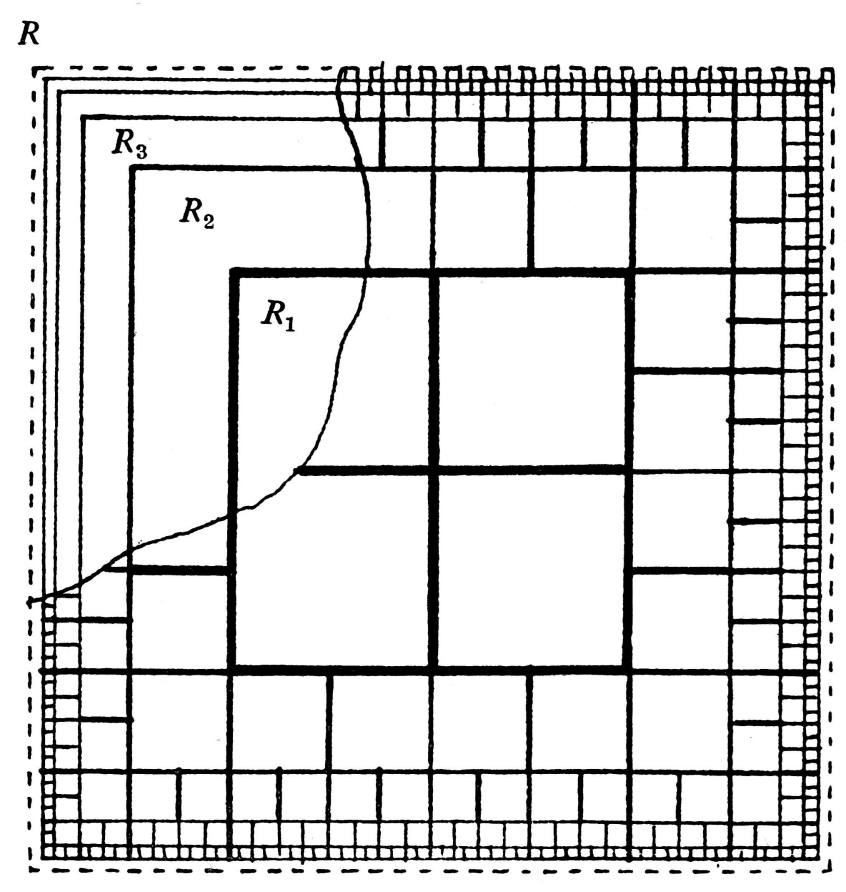

for $k=1,2,3, \ldots$. The condition (ii) implies $2 Q \subset R$ for all cubes $Q \in \bigcup_{k \geqq 1} \mathscr{F}_{k}$, while for cubes $Q \in \mathscr{F}_{k}$ it follows from v) that $|2 Q|^{-\eta} \leqq 2^{n k \eta}|R|^{-\eta}$ for $\eta=(1-\beta) p / r$. Hence by (3.2)

$$
\int_{Q}|f|^{p} \leqq C^{p} A^{p} 2^{n k \eta}|Q|^{1-\beta}\left(\int_{2 Q}|f|^{p}\right)^{\beta}\left(f_{R}|f|^{r}\right)^{\eta},
$$


where $C$ depends only on $n, p, r, s$. Summing over the cubes $Q \in \mathscr{F}_{k}$ and using i) through iv) we obtain

$$
\begin{gathered}
\int_{R_{k}}|f|^{p} \leqq C^{p} A^{p} 2^{n k \eta}\left(f_{R}|f|^{r}\right)^{\eta} \sum_{Q \in \mathscr{F}_{k}}|Q|^{1-\beta}\left(\int_{2 Q}|f|^{p}\right)^{\beta} \\
\leqq C^{p} A^{p} 2^{n k \eta}\left(f_{R}|f|^{r}\right)^{\eta}\left(\sum_{Q \in \mathscr{F}_{k}}|Q|\right)^{1-\beta}\left(\sum_{Q \in \mathscr{F}_{k}} \int_{2 Q}|f|^{p}\right)^{\beta} \\
\leqq C^{p} A^{p} 2^{n k \eta}\left(f_{R}|f|^{r}\right)^{\eta}\left|R_{k}\right|^{1-\beta} 4^{n \beta}\left(\int_{R_{k+1}}|f|^{p}\right)^{\beta} \\
\leqq C^{p} A^{p} 2^{n k \eta} 6^{n \beta}\left|R_{k}\right|\left(f_{R}|f|^{r}\right)^{\eta}\left(f_{R_{k+1}}|f|^{p}\right)^{\beta} .
\end{gathered}
$$

Next we iterate these inequalities to get

$$
f_{R_{k}}|f|^{p} \leqq\left[C^{p} A^{p} 6^{n \beta}\left(f_{R}|f|^{r}\right)^{\eta}\right]^{\alpha_{l}}\left[2^{n \eta}\right]^{\gamma_{l}}\left[f_{R_{k+l+1}}|f|^{p}\right]^{\beta^{l+1}}
$$

for each $l=0,1,2, \ldots$ and $k=1,2,3, \ldots$, where

and

$$
\alpha_{l}=\sum_{j=0}^{l} \beta^{j} \rightarrow 1 /(1-\beta)
$$

$$
\gamma_{l}=\sum_{j=0}^{l}(k+j) \beta^{j} \rightarrow(k(1-\beta)+\beta) /(1-\beta)^{2} .
$$

Letting $l \rightarrow \infty$, we obtain the inequality

$$
\begin{aligned}
f_{R_{k}}|f|^{p} & \leqq\left(C^{p} A^{p} 6^{n \beta}\right)^{1 /(1-\beta)}\left(f_{R}|f|^{r}\right)^{p / r} 2^{n k p / r} 2^{n p \beta / r(1-\beta)} \\
& \leqq C^{\prime}(n, p, r, s) A^{1 /(1-\beta)} 2^{n k / r}\left(f_{R}|f|^{r}\right)^{1 / r}
\end{aligned}
$$

for every cube $R \subset \Omega$ and $k=1,2, \ldots$. Finally, if $\sigma>1$ and $Q$ is a cube with $\sigma Q \subset \Omega$, we apply the last inequality with $R=\sigma Q$ and $k$ such that $1-2^{1-k}<$ $\sigma^{-1}<1-2^{-k}$. Then $Q \subset R_{k}, 2^{n k / r} \leqq(2 \sigma /(\sigma-1))^{n / r}$ and

$$
\left(f_{Q}|f|^{p}\right)^{1 / p} \leqq C(n, p, r, s) A^{(p-r) s /(p-s) r}\left(\frac{\sigma}{\sigma-1}\right)^{n / r}\left(f_{\sigma Q}|f|^{r}\right)^{1 / r} .
$$

This proves Theorem 2 .

R emark. The theorem becomes very simple in the case when a "strong" reverse Hölder inequality is assumed, i.e., when the double cube $2 Q$ is replaced by $Q$ in (3.0). In this case Hölder's inequality leads to an inequality like (3.2) but with the cube $Q$ in place of $2 Q$ resulting in the following conclusion:

$$
\left(f_{Q}|f|^{p}\right)^{1 / p} \leqq A^{(p-r) s /(p-s) r}\left(f_{Q}|f|^{r}\right)^{1 / r}
$$




\section{Domains with a chain condition}

Definition 1. An open set $\Omega \subset \boldsymbol{R}^{n}$ is said to be a member of $\mathscr{F}(\sigma, N), \sigma>1$, $N \geqq 1$, if there exists a covering $\mathscr{V}$ of $\Omega$ consisting of open cubes such that:

i)

$$
\sum_{Q \in \mathscr{V}} \chi_{\sigma Q}(x) \leqq N \chi_{\Omega}(x), \quad x \in \boldsymbol{R}^{n}
$$

ii) There is a distinguished cube $Q_{0} \in \mathscr{V}$ (called the central cube) which can be connected with every cube $Q \in \mathscr{V}$ by a chain of cubes $Q_{0}, Q_{1}, \ldots, Q_{s}=Q$ from $\mathscr{V}$ such that for each $v=0,1, \ldots, s-1$

$$
Q \subset N Q_{v}
$$

There is a cube $R_{v} \subset \boldsymbol{R}^{n}$ (this cube does not need be a member of $\mathscr{V}$ ) such that

$$
R_{v} \subset Q_{v} \cap Q_{v+1} \text { and } Q_{v} \cup Q_{v+1} \subset N R_{v}
$$

(compare with [2]).

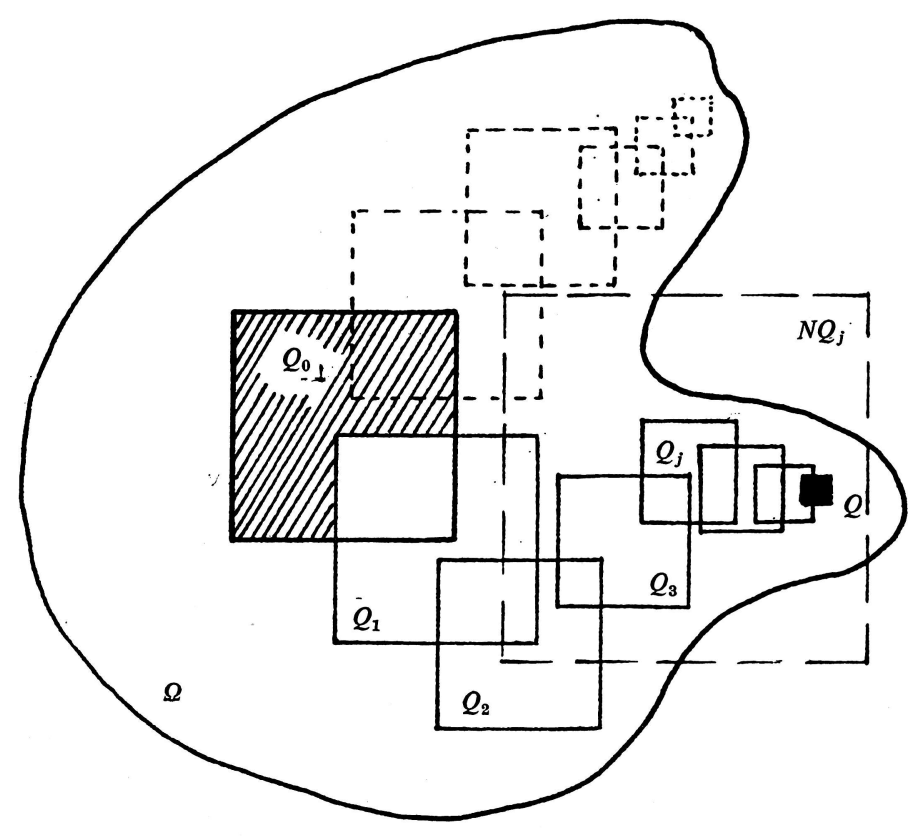

The classes $\mathscr{F}(\sigma, N)$ contain many important types of domains in $\boldsymbol{R}^{n}$, for example, cubes, balls and John domains [2]. $\mathscr{F}$-domains have no external cusps but they 
may have certain internal cusps. Clearly, if $\Omega \in \mathscr{F}(\sigma, N)$, then $\Omega \subset N Q_{0}$ is bounded. We will also be concerned with some unbounded domains. We say that $\Omega \in \mathscr{G}(\sigma, N)$ if $\Omega=\bigcup_{i=1}^{\infty} \Omega_{i}$ where $\Omega_{i} \in \mathscr{F}(\sigma, N), \sigma>1, N \geqq 1$ and $\Omega_{i} \subset \Omega_{i+1}$ for $i=1,2,3, \ldots$ The half space

$$
\mathrm{K}=\left\{\left(x_{1}, \ldots x_{n}\right) ; \quad x_{n}<0\right\}
$$

belongs to $\mathscr{G}(\sigma, N)$ for some $\sigma=\sigma(n)>1$ and $N=N(n) \geqq 1$.

The fundamental property of a domain $\Omega \in \mathscr{F}(\sigma, N)$ is that certain local estimates which are valid in cubes contained in $\Omega$ automatically also hold in $\Omega$. The following theorem illustrates this phenomenon.

Theorem 3. Let $\sigma>1, N \geqq 1,0<p<\infty, \Omega \in \mathscr{F}(\sigma, N), w \in A_{M}^{q}\left(\boldsymbol{R}^{n}\right), q>1, M \geqq 1$ and let $u$ and $v$ be measurable functions defined on $\Omega$. Suppose that for each cube $Q$ with $\sigma Q \subset \Omega$ we have

$$
\|u\|_{p, Q, \mu}^{\#} \leqq A\|v\|_{p, \sigma Q, \mu},
$$

where $d \mu(x)=w(x) d x$ and $A$ is a constant independent of $Q$. Then

$$
\|u\|_{p, \Omega, \mu}^{\#} \leqq B\|v\|_{p, \Omega, \mu},
$$

where $B$ depends only on $n, p, \sigma, N, q, M$ and $A$.

The proof is very similar to that in the paper by J. Boman [2]. We give this argument for the sake of completeness.

Proof. We use the notation and the covering $\mathscr{V}$ described in Definition 1. We begin with the following properties of the measure $d \mu(x)=w(x) d x$; since $w \in A_{M}^{q}\left(\boldsymbol{R}^{n}\right)$,

$$
\mu(N Q) \leqq M N^{n q} \mu(Q)
$$

for each cube $Q \subset \boldsymbol{R}^{n}$ by (2.5) and

$$
\max \left(\mu\left(Q_{v}\right), \mu\left(Q_{v+1}\right)\right) \leqq M N^{n q} \mu\left(Q_{v} \cap Q_{v+1}\right)
$$

for the sequence of cubes $Q_{v}, Q_{v+1}, v=0,1, \ldots, s-1$ described in ii).

Now inequality (4.0) says that for each $Q \in \mathscr{V}$ there is a real number $a_{Q}$ such that

$$
\left\|u-a_{Q}\right\|_{p, Q, \mu} \leqq A\|v\|_{p, \sigma Q, \mu} .
$$

Hereafter we will use the following elementary inequality $|a+b|^{p} \leqq 2^{p}\left(|a|^{p}+|b|^{p}\right)$ for all $p>0$. In particular we have

$$
\int_{\Omega}\left|u-a_{Q_{0}}\right|^{p} d \mu \leqq 2^{p} \sum_{Q \in \mathcal{V}} \int_{Q}\left|u-a_{Q}\right|^{p} d \mu+2^{p} \sum_{Q \in \mathcal{V}} \int_{Q}\left|a_{Q_{0}}-a_{Q}\right|^{p} d \mu .
$$

The first sum is estimated by (4.4) and the condition i)

$$
\sum_{Q \in \mathcal{V}} \int_{Q}\left|u-a_{Q}\right|^{p} d \mu \leqq A^{p} \sum_{Q \in \mathscr{V}} \int_{\sigma Q}|v|^{p} d \mu \leqq N A^{p} \int_{\Omega}|v|^{p} d \mu .
$$


The second sum in (4.5) requires deeper arguments to estimate in terms of the integral $\int_{\Omega}|v|^{p} d \mu$.

Fix a cube $Q=Q_{s} \in \mathscr{V}$ and let $\left\{Q_{v}\right\}, v=0,1, \ldots, s$, be the chain referred to in ii) and write $a_{v}=a_{Q_{v}}$. We have

$$
\left|a_{Q_{0}}-a_{Q}\right| \leqq \sum_{v=0}^{s-1}\left|a_{v}-a_{v+1}\right| \text {. }
$$

Now by (4.3) and (4.4) we have

$$
\begin{aligned}
\left|a_{v}-a_{v+1}\right|^{p}= & f_{Q_{v} \cap Q_{v+1}}\left|a_{v}-a_{v+1}\right|^{p} d \mu \leqq \sum_{\alpha=v, v+1} 2^{p} M N^{n q} f_{Q_{\alpha}}\left|u-a_{\alpha}\right|^{p} d \mu \\
& \leqq 2^{p} A^{p} M N^{n q} \sum_{\alpha=v, v+1} \frac{1}{\mu\left(Q_{\alpha}\right)} \int_{\sigma Q_{\alpha}}|v|^{p} d \mu .
\end{aligned}
$$

Since $Q \subset N Q_{\alpha}$ for $\alpha=v, v+1,0 \leqq v \leqq s-1$ (see ii)),

and by (4.7)

$$
\left|a_{v}-a_{v+1}\right|^{p} \chi_{Q}(x) \leqq 2^{p} A^{p} M N^{n q} \sum_{\alpha=v, v+1} \frac{\chi_{N Q_{\alpha}}(x)}{\mu\left(Q_{\alpha}\right)} \int_{\sigma Q_{\alpha}}|v|^{p} d \mu
$$

$$
\left|a_{Q_{0}}-a_{Q}\right| \chi_{Q}(x) \leqq 2 \cdot 2 \cdot 2^{1 / p} A M^{1 / p} N^{n q / p} \sum_{R \in \mathcal{V}}\left(\frac{1}{\mu(R)} \int_{\sigma R}|v|^{p} d \mu\right)^{1 / p} \chi_{N R}(x)
$$

for every $x \in \boldsymbol{R}^{n}$. Hence

$$
\begin{gathered}
\sum_{Q \in \mathcal{V}} \int_{Q}\left|a_{Q_{0}}-a_{Q}\right|^{p} d \mu \\
\leqq 2^{2 p+1} A^{p} M N^{n q+1} \int_{R^{n}}\left|\sum_{R \in \mathcal{V}}\left(\frac{1}{\mu(R)} \int_{\sigma R}|v|^{p} d \mu\right)^{1 / p} \chi_{N R}(x)\right|^{p} d \mu(x) .
\end{gathered}
$$

If $0<p \leqq 1$ we use the inequality $\left|\Sigma_{\alpha} t_{\alpha}\right|^{p} \leqq \Sigma_{\alpha}\left|t_{\alpha}\right|^{p}$, (4.2) and the condition i) to get

$$
\begin{aligned}
& \sum_{Q \in \mathcal{V}} \int_{Q}\left|a_{Q_{0}}-a_{Q}\right|^{p} d \mu \leqq 2^{2 p+1} A^{p} M N^{n q+1} \sum_{R \in \mathscr{V}} \frac{\mu(N R)}{\mu(R)} \int_{\sigma R}|v|^{p} d \mu \\
& \leqq 2^{2 p+1} A^{p} M^{2} N^{2 n q+1} \sum_{R \in \mathscr{V}} \int_{\sigma R}|v|^{P} d \mu \leqq 2^{2 p+1} A^{p} M^{2} N^{2 n q+2} \int_{\Omega}|v|^{p} d \mu .
\end{aligned}
$$

This, (4.5) and (4.6) give the result for $0<p \leqq 1$. In the case $1 \leqq p<\infty$, we use the following lemma, similar to one of the Strömberg-Torchinsky [12], to estimate the right hand side of (4.8).

Lemma 4. If $\mathscr{V}$ is an arbitrary collection of cubes in $\boldsymbol{R}^{n}$ and $A_{Q}$ are non-negative numbers associated with the cubes $Q \in \mathscr{V}$ and $w \in A_{M}^{q}\left(\boldsymbol{R}^{n}\right), d \mu(x)=w(x) d x$, then for $1 \leqq p<\infty$ and $N \geqq 1$ we have

$$
\left\|\sum_{Q \in \mathscr{V}} A_{Q} \chi_{N Q}\right\|_{p, R^{n}, \mu} \leqq B_{p}\left\|\sum_{Q \in \mathscr{V}} A_{Q} \chi_{Q}\right\|_{p, R^{n}, \mu},
$$

where $B_{p}$ also depends on $n, q, N$ and $M$ but this is independent of the collection $\mathscr{V}$ and the numbers $A_{Q}$.

This lemma is proved as in [2] by use of the weighted maximal theorem [3]. 
Now, returning from this lemma to (4.8) we get

$$
\begin{gathered}
\sum_{\Omega \in \mathscr{V}} \int_{Q}\left|a_{Q_{0}}-a_{Q}\right|^{p} d \mu \\
\leqq 2^{2 p+1} A^{p} M N^{n q+1} B_{2}^{2} \int_{R^{n}}\left|\sum_{R \in \mathscr{V}}\left(\frac{1}{\mu(R)} \int_{\sigma R}|v|^{p} d \mu\right)^{1 / p} \chi_{R}(x)\right|^{p} d \mu(x) .
\end{gathered}
$$

Since

$$
\sum_{R \in \mathscr{V}} \chi_{R}(x) \leqq \sum_{R \in \mathscr{V}} \chi_{\sigma R}(x) \leqq N \chi_{\Omega}(x),
$$

with the elementary inequality

we get

$$
\left|\sum_{v=1}^{N} t_{v}\right|^{p} \leqq N^{p-1} \sum_{v=1}^{N}\left|t_{v}\right|^{p}
$$

$$
\begin{gathered}
\sum_{Q \in \mathcal{V}} \int_{Q}\left|a_{Q_{0}}-a_{Q}\right|^{p} d \mu \leqq 2^{2 p+1} A^{p} M N^{p+n q} B_{p}^{p} \sum_{R \in \mathscr{V}} \int_{\sigma R}|v|^{p} d \mu \\
\leqq 2^{2 p+1} A^{p} M N^{p+n q+1} B_{p}^{p} \int_{\Omega}|v|^{p} d \mu
\end{gathered}
$$

by the condition i). This completes the proof of Theorem 3 .

\section{Basic local estimates for quasiregular mappings}

Definition 2. Suppose $f: \Omega \rightarrow \boldsymbol{R}^{n}$ is in the Sobolev space $W_{n, \mathrm{loc}}^{1}(\Omega)$. Then $f$ is said to be $K$-quasiregular $(K$-qr), $1 \leqq K<\infty$, if

$$
|D f(x)|^{n} \leqq K J_{f}(x)
$$

for almost every $x \in \Omega$; here $D f(x): \boldsymbol{R}^{n} \rightarrow \boldsymbol{R}^{n}$ is the linear tangent map (or the Jacobian matrix) with norm $|D f(x)|$ and Jacobian determinant $J_{f}(x)$.

Quasiregular mappings were introduced by Ju. Rešetnjak [11] and O. Martio, S. Rickman and J. Väisälä [8], [9] in the 1960's. They serve as generalizations of both holomorphic functions of one complex variable and quasiconformal mappings in $\boldsymbol{R}^{n}$. When $n=2, f$ is $K$-qr if and only if it is a holomorphic function. The definition above originates from an analytic characterization of $K$-quasiconformal maps due to F. W. Gehring [4].

The components $f^{1}, f^{2}, \ldots, f^{n}$ of a $K$-qr map are related by some algebraic inequalities. One of them is the inequality

$$
\left|\nabla f^{i}(x)\right| \leqq K\left|\nabla f^{j}(x)\right|
$$

for almost every $x \in \Omega$ and $i, j=1,2, \ldots, n$. In fact, for every $k=1,2, \ldots, n,\left|\nabla f^{k}(x)\right| \leqq$ $|D f(x)|$ and by Hadamard inequality $\left|\nabla f^{1}\right|\left|\nabla f^{2}\right| \ldots\left|\nabla f^{n}\right| \geqq J_{f}$. Hence

for $i, j=1,2, \ldots, n$.

$$
\left|\nabla f^{i}\right|=\frac{\left|\nabla f^{i}\right| \prod_{j \neq k=1}^{n}\left|\nabla f^{k}\right|}{\prod_{k=1}^{n}\left|\nabla f^{\cdot k}\right|}\left|\nabla f^{j}\right| \leqq \frac{|D f|^{n}}{J_{f}}\left|\nabla f^{j}\right| \leqq K\left|\nabla f^{j}\right|
$$


If $f=u+i v$ is analytic, then $u$ and $v$ satisfy the Cauchy-Riemann system $u_{x}=v_{y}$, $v_{x}=-u_{y}$ and the gradients $\nabla u$ and $\nabla v$ are perpendicular vectors of equal length. The above gradient estimates partially explain the result of Hardy and Littlewood. To eliminate the first derivatives in the estimates of the components of a quasiregular mapping we will use the following general estimation of the Jacobian determinant:

$$
\left|\int_{\Omega} \varphi^{n}(x) J_{f}(x) d x\right| \leqq n\left(\int_{\Omega}\left|f^{j} \nabla \varphi\right|^{n}\right)^{1 / n}\left(\int_{\Omega}|\varphi D f|^{n}\right)^{(n-1) / n}
$$

This holds for every mapping $f=\left(f^{1}, \ldots, f^{n}\right)$ from the space $W_{n, \text { loc }}^{1}(\Omega)$, for each $j=1,2, \ldots, n$ and for each test function $\varphi \in C_{0}^{\infty}(\Omega), \varphi \geqq 0$. We sketch the proof of (5.2) below. If $f \in C^{\infty}(\Omega)$, then by Stokes' formula and Hölder's inequality we have

Hence

$$
\begin{gathered}
\varphi^{n} J_{f} d x=d f^{1} \wedge \ldots \wedge d f^{j-1} \wedge \varphi^{n} d f^{j} \wedge d f^{j+1} \wedge \ldots \wedge d f^{n} \\
=d\left(\varphi^{n} f^{j} d f^{1} \wedge \ldots \wedge d f^{j-1} \wedge d f^{j+1} \wedge \ldots \wedge d f^{n}\right) \\
-f^{j} d f^{1} \wedge \ldots \wedge d f^{j-1} \wedge d \varphi^{n} \wedge d f^{j+1} \wedge \ldots \wedge d f^{n}
\end{gathered}
$$

$$
\begin{aligned}
\left|\int_{\Omega} \varphi^{n} J_{f}\right| & \leqq \int_{\Omega}\left|f^{j}\right|\left|\nabla \varphi^{n}\right||D f|^{n-1}=n \int_{\Omega}\left|f^{j} \nabla \varphi\right||\varphi D f|^{n-1} \\
& \leqq n\left(\int_{\Omega}\left|f^{j} \nabla \varphi\right|^{n}\right)^{1 / n}\left(\int_{\Omega}|\varphi D f|^{n}\right)^{(n-1) / n} .
\end{aligned}
$$

The general case then follows by an approximation argument.

Now if $f$ is $K$-qr, then for each $\varphi \in C_{0}^{\infty}(\Omega), \varphi \geqq 0$, we have

for $j=1,2, \ldots, n$.

$$
\left(\int_{\Omega}|\varphi D f|^{n}\right)^{1 / n} \leqq n K\left(\int_{\Omega}\left|f^{j} \nabla \varphi\right|^{n}\right)^{1 / n}
$$

Next we apply this inequality to a test function $\varphi \in C_{0}^{\infty}(\sigma \Omega), \varphi \geqq 0, \varphi(x) \geqq 1$ on $Q$ and

$$
|\nabla \varphi(x)| \leqq \frac{C(n)}{(\sigma-1) \operatorname{diam} Q}
$$

where $\sigma>1$ and $Q$ is a cube with $\sigma Q \subset \Omega$; clearly such a function exists. We then get

$$
\left(f_{Q}|D f|^{n}\right)^{1 / n} \leqq \frac{C(n) \sigma K}{\sigma-1}\left(f_{\sigma Q}\left|f^{j}\right|^{n}\right)^{1 / n}
$$

for $j=1,2, \ldots, n$. Finally by the Poincaré-Sobolev inequality (2.2)

$$
\left(f_{Q}\left|f^{i}-f_{Q}^{i}\right|^{p}\right)^{1 / p} \leqq C_{1}\left(f_{Q}\left|D f^{n}\right|\right)^{1 / n} \leqq \frac{C(n, p) \sigma K}{\sigma-1}\left(f_{\sigma Q}\left|f^{j}\right|^{n}\right)^{1 / n}
$$

for $i, j=1,2, \ldots, n, p>0$ and the cube $Q$ with $\sigma Q \subset \Omega$. This inequality with $p>n$, 
$\sigma=2$ and $i=j$ reduces to the "weak" reverse Hölder inequality

$$
\left(f_{Q}\left|f^{j}\right|^{p}\right)^{1 / p} \leqq 4 C(n, p) K\left(f_{2 Q}\left|f^{j}\right|^{n}\right)^{1 / n}
$$

for $j=1,2, \ldots, n$ and each cube $Q$ with $2 Q \subset \Omega$.

Now we are ready to prove the following local results.

Proposition 1. Let $f=\left(f^{1}, f^{2}, \ldots, f^{n}\right)$ be $K$-qr in $\Omega$ and let $\sigma>1,0<p<\infty$, $0<r<\infty$. Then

and

$$
\left(f_{Q}\left|f^{j}\right|^{p}\right)^{1 / p} \leqq B_{1}\left(f_{\sigma Q}\left|f^{j}\right|^{r}\right)^{1 / r}
$$

$$
\left(f_{Q}\left|f^{i}-f_{Q}^{i}\right|^{p}\right)^{1 / p} \leqq B_{2}\left(f_{\sigma Q}\left|f^{j}\right|^{r}\right)^{1 / r}
$$

for $i, j=1,2, \ldots, n$ and cubes $Q$ with $\sigma Q \subset \Omega$. Here the constants $B_{1}=B_{1}(\sigma)$ and $B_{2}=B_{2}(\sigma)$ depend only on $\sigma, n, p, r$, and $K$.

Proof. We may assume without loss of generality that $p>n$. Inequality (5.6) follows from (5.5) and Theorem 2. To get (5.7) we apply (5.4) with the factor $\sqrt{\sigma}$ in place of $\sigma$ and after this we apply (5.6) with $p=n, \sqrt{\sigma}$ in place of $\sigma$ and with the cube $\sqrt{\sigma} Q$ in place of $Q$. We then obtain

$$
\begin{aligned}
& \left(f_{Q}\left|f^{i}-f_{Q}^{i}\right|^{p}\right)^{1 / p} \leqq \frac{C(n, p) \sqrt{\sigma} K}{\sqrt{\sigma}-1}\left(f_{\sqrt{\sigma} Q}\left|f^{j}\right|^{n}\right)^{1 / n} \\
& \leqq \frac{c(n, p) B_{1}(\sqrt{\sigma}) \sqrt{\sigma} K}{\sqrt{\sigma}-1}\left(f_{\sigma Q}\left|f^{j}\right|^{r}\right)^{1 / r}
\end{aligned}
$$

This proves (5.7).

The weighted version of (5.7) reads as follows.

Proposition 2. If $f=\left(f^{1}, f^{2}, \ldots, f^{n}\right)$ is $K-q r$ in $\Omega$ and if $w \in A_{M}^{q}\left(\boldsymbol{R}^{n}\right), q>1$, $M \geqq 1, d \mu(x)=w(x) d x$, then

$$
\left\|f^{i}\right\|_{p, \Omega, \mu}^{\#} \leqq B_{3}(\sigma)\left\|f^{j}\right\|_{p, \sigma Q, \mu}
$$

for $i, j=1,2, \ldots, n, 0<p<\infty, \sigma>1$ and each cube $Q$ with $\sigma Q \subset \Omega$. Here $B_{3}(\sigma)$ depends also on $n, p, q, M$ and $K$.

Proof. Since $w \in A_{M}^{q}\left(\boldsymbol{R}^{n}\right)$, we have the reverse Hölder inequality (2.6) with some $\beta>1$. By (5.7) we get

$$
\begin{gathered}
\left(f_{Q}\left|f^{i}-f_{Q}^{i}\right|^{p} w\right)^{1 / p} \leqq\left(f_{Q} w^{\beta}\right)^{1 / \beta p}\left(f_{Q}\left|f^{i}-f_{Q}^{i}\right|^{p \beta /(\beta-1)}\right)^{(\beta-1) / \beta p} \\
\leqq C_{3}^{1 / p} B_{2}\left(f_{Q} w\right)^{1 / p}\left(f_{\sigma Q} \mid f^{j \mid p / q}\right)^{q / p}=C_{3}^{1 / p} B_{2}|Q|^{-1 / p} \mu(Q)^{1 / p}\left(f_{\sigma Q}\left|f^{j}\right|^{p / q}\right)^{q / p} .
\end{gathered}
$$


On the other hand, by Hölder's inequality and the $A_{M}^{q}$-condition (2.4) we have

$$
\begin{gathered}
\left(f_{\sigma Q} \mid f^{j \mid p / q}\right)^{q / p} \leqq\left(f_{\sigma Q}\left|f^{j}\right|^{p} w\right)^{1 / p}\left(f_{\sigma Q} w^{1 /(1-q)}\right)^{(q-1) / p} \\
\leqq M^{1 / p}\left(f_{\sigma Q} w\right)^{-1 / p}\left(f_{\sigma Q}\left|f^{j}\right|^{p} w\right)^{1 / p} \leqq M^{1 / p}|\sigma Q|^{1 / p} \mu(Q)^{-1 / p}\left\|f^{j}\right\|_{p, \sigma Q, \mu} .
\end{gathered}
$$

Thus we obtain (5.8) with $B_{3}=C_{3}^{1 / p} B_{2} M^{1 / p} \sigma^{n / p}$.

As solutions of certain equations of elliptic type, the components of a $K$-qr map have many properties similar to those of harmonic functions. We conclude this section with one such result.

Proposition 3. Let $f$ be $K$-qr in a cube $Q$ centered at $x_{0}$. Then for each $r>0$

$$
\left|f\left(x_{0}\right)\right| \leqq B_{4}(n, r, K)\left(f_{Q}|f|^{r}\right)^{1 / r} .
$$

Proof. We will use the following form of Gehring's well-known $L^{p}$-result (see [1], Theorem 5.1, p. 285): There exists an exponent $s=s(n, K)>n$ such that

$$
\|D f\|_{F, s} \leqq C(n, s, K)(\operatorname{dist}(F, \partial \Omega))^{n / s-1}\|D f\|_{\Omega, n}
$$

for every compact $F \subset \Omega$. Now by Sobolev's inequality (2.3), (5.10) and (5.3), for every cube $R$ centered at $x_{0}$ with $4 R \subset Q$ we have

$$
\begin{aligned}
& \left|f\left(x_{0}\right)\right| \leqq C_{2}\left[\left(f_{R}|f|^{s}\right)^{1 / s}+\operatorname{diam} R\left(f_{R}|D f|^{s}\right)^{1 / s}\right] \\
& \leqq C(n, s, K)\left[\left(f_{R}|f|^{s}\right)^{1 / s}+\left(\int_{2 R}|D f|^{n}\right)^{1 / n}\right] \\
& \leqq C^{\prime}(n, s, K)\left[\left(f_{R}|f|^{s}\right)^{1 / s}+\left(f_{3 R}|f|^{n}\right)^{1 / n}\right] .
\end{aligned}
$$

Finally, by (5.6),

and

$$
\left(f_{R}|f|^{s}\right)^{1 / s} \leqq B_{1}\left(f_{4 R}|f|^{r}\right)^{1 / r}
$$

$$
\left(f_{3 R}|f|^{n}\right)^{1 / n} \leqq B_{1}\left(f_{4 R}|f|^{r}\right)^{1 / r}
$$

All together these inequalities imply (5.9) with $Q=4 R$.

\section{Global estimates and their consequences}

If we apply Proposition 2 and Theorem 3 to $f-a$ we obtain

Theorem 4. Let $\Omega \in \mathscr{F}(\sigma, N), 0<p<\infty$ and $w \in A_{M}^{q}\left(\boldsymbol{R}^{n}\right)$. If $f=\left(f^{1}, f^{2}, \ldots, f^{n}\right)$ is $K$-qr in $\Omega$, then

$$
\left\|f^{i}\right\|_{p, \Omega, \mu}^{\#} \leqq A_{p}\left\|f^{j}\right\|_{p, \Omega, \mu}^{\#}
$$


for $i, j=1,2, \ldots, n$, where $d \mu(x)=w(x) d x$ and $A_{p}$ depends also on $n, K, \sigma, N, q$ and $M$.

Since cubes are in $\mathscr{F}(\sigma, N)$, this theorem implies

Corollary 1. Let $\Omega$ be an arbitrary open set in $\boldsymbol{R}^{n}$. If $f=\left(f^{1}, \ldots, f^{n}\right)$ is $K$-qr in $\Omega$ and $w \in A_{M}^{q}\left(\boldsymbol{R}^{n}\right)$, then

$$
M_{p}^{\#}\left(f^{i}, \mu\right)(x) \leqq A_{p} M_{p}^{\#}\left(f^{j}, \mu\right)(x)
$$

for all $x \in \Omega, 0<p<\infty$ and $i, j=1,2, \ldots, n . A_{p}$ is the constant that appears in (6.0) when $\Omega$ is a cube. Thus $A_{p}$ depends on $n, K, q$ and $M$ but not on $\Omega$.

Corollary 2. With the same assumptions as in Corollary 1 we have

$$
\left\|f^{i}\right\|_{\Omega, \mu}^{\mathrm{BMO}} \leqq A_{1}\left\|f^{j}\right\|_{\Omega, \mu}^{\mathrm{BMO}}
$$

for $i, j=1,2, \ldots, n$, where $A_{1}=A_{1}(n, K, q, M)$ is independent of the domain $\Omega$.

As concerns unbounded domains, we have the following extension of Theorem 4 which follows from Lemma 1.

Corollary 3. Let $\Omega \in \mathscr{G}(\sigma, N)$ and $w \in A_{M}^{q}\left(\boldsymbol{R}^{n}\right)$. If $f=\left(f^{1}, f^{2}, \ldots, f^{n}\right)$ is $K$-qr in $\Omega$ such that $f^{j} \in L^{p}(\Omega, \mu), 0<p<\infty$, for some $1 \leqq j \leqq n$, then for each $i=1,2, \ldots, n$

$$
\left\|f^{i}\right\|_{p, \Omega, \mu}^{\#} \leqq A_{p}\left\|^{j}\right\|_{p, \Omega, \mu}^{\#} .
$$

In contrast to this result we have

Example. Let $\Omega \subset \boldsymbol{R}^{2}$ be the half strip:

$$
\Omega=\{z=x+i y ; 1<x<\infty, 0<y<1\} .
$$

Consider the analytic function $f(z)=\log z=\log |z|+i \theta$ in $\Omega$. For $p>1$

while

$$
\int_{\Omega} \theta^{p} d x d y \leqq \int_{\Omega}(\tan \theta)^{p} d x d y=\frac{1}{p^{2}-1}
$$

$$
\int_{\Omega}|\log | z||^{p} d x d y \geqq \int_{1}^{\infty}(\log t)^{p} d t=\infty .
$$

Corollary 4. Let $\Omega=g(\mathrm{H})$ or $g\left(\boldsymbol{B}^{n}\right)$ where $\boldsymbol{B}^{n}$ is the unit ball in $\boldsymbol{R}^{n}$ and $g: \boldsymbol{R}^{n} \rightarrow \boldsymbol{R}^{n}$ is a quasiconformal mapping. If $f=\left(f^{1}, \ldots, f^{n}\right)$ is $K$-qr in $\Omega$, then

$$
\left\|f^{i}\right\|_{p, \Omega}^{\#} \leqq A_{p}\left\|f^{j}\right\|_{p, \Omega}^{\#}
$$

for $0<p<\infty, i, j=1,2, \ldots, n$, where $A_{p}$ depends on $n, K$ and the dilatation of $g$.

This corollary is a direct consequence of the previous weighted estimates by change of variables $x=g\left(x^{\prime}\right)$. Recall that $J_{g}\left(x^{\prime}\right)$ belongs to the Muckenhoupt class $A_{M}^{q}\left(\boldsymbol{R}^{n}\right)$ for some $q>1$ and $M \geqq 1$ depending only on $n$ and the dilatation of $g$. As a consequence of the study of the weighted inequalities for general quasiregular mappings we also get the following generalization of Theorem 1. 
Corollary 5. If $f=u+i v$ is analytic in a quasidisk $D \subset R^{2}$ then

$$
\|u\|_{p, D}^{\#} \leqq A_{p}(D)\|v\|_{p, D}^{\#}
$$

for every $0<p<\infty$.

Finally, we return to inequality (6.3) with $\Omega$ the upper-half space $K$ and $d \mu(x)=d x$. Then it is easy to see that the constants $a_{1}, a_{2}, \ldots, a_{n}$ for which

$$
\left\|f^{i}-a_{i}\right\|_{p, \mathbf{K}} \leqq A_{p}(n, K)\left\|f^{j}\right\|_{p, \mathrm{~K}}
$$

are uniquely determined. Indeed, the vector $a=\left(a_{1}, a_{2}, \ldots, a_{n}\right)$ is the non-tangential limit of $f$ at infinity. More precisely,

Proposition 4. Let $f=\left(f^{1}, f^{2}, \ldots, f^{n}\right)$ be $K$-qr in K. Assume that one of the components, say $f^{j}, 1 \leqq j \leqq n$, belongs to $L^{p}(\mathrm{~K}), 0<p<\infty$. Then there is a constant vector $a=f(\infty)$ such that $f-f(\infty) \in L^{p}(\mathrm{~K})$ and

$$
\|f-f(\infty)\|_{p, \mathrm{~K}} \leqq A_{p}(n, K)\left\|f^{j}\right\|_{p, \mathrm{~K}} .
$$

Moreover,

$$
\sup _{x \in \mathrm{K}} x_{n}^{n / p}|f(x)-f(\infty)| \leqq c_{p}(n, K)\left\|f^{j}\right\|_{p, \mathrm{~K}} .
$$

Proof. The statement (6.7) follows from (6.6). To prove (6.8) fix a point $x=\left(x_{1}, x_{2}, \ldots, x_{n}\right) \in \mathrm{K}$ and consider the cube $Q \subset \mathrm{K}$ centered at $x$ and with sidelength $2 x_{n}>0$. Then by Proposition 3 applied to the map $f-f(\infty)$ we get

This proves (6.8).

$$
\begin{aligned}
|f(x)-f(\infty)| \leqq & B_{4}\left(f_{Q}|f-f(\infty)|^{p}\right)^{1 / p} \leqq B_{4}\left(2 x_{n}\right)^{-n / p}\|f-f(\infty)\|_{p, \mathrm{~K}} \\
& \leqq 2^{-n / p} B_{4} A_{p}(n, K)\left\|f^{j}\right\|_{p, \mathrm{~K}} x_{n}^{-n / p}
\end{aligned}
$$

\section{References}

[1] Bojarski, B., and T. IwANIEC: Analytical foundations of the theory of quasiconformal mappings in $\boldsymbol{R}^{n}$. - Ann. Acad. Sci. Fenn. Ser A I Math. 8, 1984. 257-324.

[2] Boman, J.: $L^{p}$-estimates for very strong elliptic systems. - Preprint, to appear in J. Analyse Math.

[3] Coifman, R. R., and C. Fefferman: Weighted norm inequalities for maximal functions and singular integrals. - Studia Math. 51, 1974, 241-250.

[4] Gehring, F. W.: Rings and quasiconformal mappings in space. - Proc. Nat. Acad. Sci. U.S.A. 47, 1961, 98-105.

[5] Gehring, F. W.: The $L^{p}$-integrability of the partial derivatives of a quasiconformal mapping. - Acta Math. 130, 1973, 265-277.

[6] Gilbarg, D., and N. S. Trudinger: Elliptic partial differential equations of second order. - Springer-Verlag, Berlin-Heidelberg-New York, 1977.

[7] Hardy, G. H., and J. E. LitTlewood: Some properties of conjugate functions. - J. Reine Angew. Math. 167, 1932, 405-423.

[8] Martio, O., S. Rickman, and J. VÄIsÄLÄ: Definitions for quasiregular mappings. - Ann. Acad. Sci. Fenn. Ser. A I Math. 448, 1969, 1-40. 
[9] Martio, O., S. RICKMAN, and J. VÄıÏ̈̈̈̈: Topological and metric properties of quasiregular mappings. - Ann. Acad. Sci. Fenn. Ser. A I Math. 488, 1971, 1-31.

[10] Muckenhioupt, B.: Weighted norm inequalities for the Hardy maximal function. - Trans. Amer. Math. Soc. 165, 1972, 207-226.

[11] REŠETNJAK, JU. G.: Estimates of the modulus of continuity for certain mappings. - Sibirsk. Math. Ž. 7, 1966, 1106-1114 (Russian).

[12] Strömberg, J. O., and A. TorchinsKy: Weights, sharp maximal functions and Hardy spaces. - Bull. Amer. Math. Soc. (N. S.) 3, 1980, 1053-1056.

Polish Academy of Sciences Institute of Mathematics

P. O. Box 137

00-950 Warsaw

Poland

Received 15 June 1984
University of Michigan

Department of Mathematics

Ann Arbor, MI 48109

USA. 